\title{
The impact of obesity and bariatric surgery on the immune microenvironment of the endometrium
}

\author{
Anie Naqvi ${ }^{1}$, Michelle L. MacKintosh ${ }^{2}$, Abigail E. Derbyshire ${ }^{2}$, Anna-Maria Tsakiroglou ${ }^{3}$, Thomas D. J. Walker (D) ${ }^{4}$, Rhona J. McVey ${ }^{5}$, \\ James Bolton ${ }^{5}$, Martin Fergie (iD) ${ }^{6}$, Steven Bagley (D) $^{7}$, Garry Ashton ${ }^{7}$, Philip W. Pemberton ${ }^{8}$, Akheel A. Syed ${ }^{9,10}$, Basil J. Ammori ${ }^{10,11}$, \\ Richard Byers ${ }^{3,5}$ and Emma J. Crosbie (iD ${ }^{2,4}$ 凶U
}

(c) The Author(s) 2021

BACKGROUND: The incidence of endometrial cancer is rising in parallel with the obesity epidemic. Obesity increases endometrial cancer risk and weight loss is protective, but the underlying mechanisms are incompletely understood. We hypothesise that the immune microenvironment may influence susceptibility to malignant transformation in the endometrium. The aim of this study was to measure the impact of obesity and weight loss on the immunological landscape of the endometrium.

METHODS: We conducted a prospective cohort study of women with class III obesity (body mass index, BMI $\geq 40 \mathrm{~kg} / \mathrm{m}^{2}$ ) undergoing bariatric surgery or medically-supervised low-calorie diet. We collected blood and endometrial samples at baseline, and two and 12 months after weight loss intervention. Serum was analysed for inflammatory markers CRP, IL-6 and TNF-a. Multiplex immunofluorescence was used to simultaneously identify cells positive for immune markers CD68, CD56, CD3, CD8, FOXP3 and PD1 in formalin-fixed paraffin-embedded endometrial tissue sections. Kruskal-Wallis tests were used to determine whether changes in inflammatory and immune biomarkers were associated with weight loss.

RESULTS: Forty-three women with matched serum and tissue samples at all three time points were included in the analysis. Their median age and BMI were 44 years and $52 \mathrm{~kg} / \mathrm{m}^{2}$, respectively. Weight loss at 12 months was greater in women who received bariatric surgery $(n=37$, median $63.3 \mathrm{~kg})$ than low-calorie diet $(n=6$, median $12.8 \mathrm{~kg})$. There were significant reductions in serum $\operatorname{CRP}\left(p=3.62 \times 10^{-6}, r=0.570\right)$ and IL-6 $(p=0.0003, r=0.459)$, but not TNF-a levels, with weight loss. Tissue immune cell densities were unchanged except for CD8+ cells, which increased significantly with weight loss $(p=0.0097, r=-0.323)$. Tissue CD3+ cell density correlated negatively with systemic IL-6 levels ( $p=0.0376 ; r=-0.318$ ).

CONCLUSION: Weight loss is associated with reduced systemic inflammation and a recruitment of protective immune cell types to the endometrium, supporting the concept that immune surveillance may play a role in endometrial cancer prevention.

International Journal of Obesity (2022) 46:605-612; https://doi.org/10.1038/s41366-021-01027-6

\section{INTRODUCTION}

Endometrial cancer is the most common gynaecological malignancy in the United Kingdom and its incidence is rising [1]. Obesity is a major risk factor for the disease, with every $5 \mathrm{~kg} / \mathrm{m}^{2}$ increase in body mass index (BMI) conferring a 1.6-fold higher risk [2]. Women with a BMI $>40 \mathrm{~kg} / \mathrm{m}^{2}$ are particularly vulnerable, with lifetime risks as high as $10-15 \%$ [3]. With $58 \%$ of the world population estimated to be overweight or obese by the year 2030
[4], the burden of endometrial cancer is set to continue [5]. Whilst most are detected early and cured by hysterectomy, women with high risk or late stage endometrial cancer have poor outcomes [6]. An improved understanding of the biological mechanisms underpinning obesity-associated endometrial cancer could lead to new targets for prevention and treatment [7]. Obesity is thought to drive endometrial carcinogenesis through adiposederived oestrogen, which, when unopposed by progesterone in

\footnotetext{
${ }^{1}$ The University of Manchester Medical School, Oxford Road, Manchester M13 9PL, UK. ${ }^{2}$ Division of Gynaecology, St Mary's Hospital, Manchester University NHS Foundation Trust, Manchester Academic Health Science Centre, Oxford Road, Manchester M13 9WL, UK. ${ }^{3}$ Division of Cancer Sciences, School of Medical Sciences, Faculty of Biology, Medicine and Health, University of Manchester, Stopford Building, Oxford Road, Manchester M13 9PT, UK. ${ }^{4}$ Gynaecological Oncology Research Group, Division of Cancer Sciences, School of

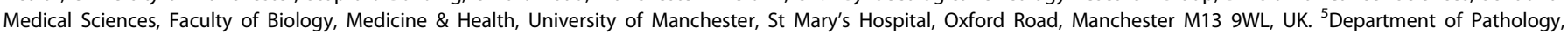
Manchester University NHS Foundation Trust, Manchester Academic Health Science Centre, Oxford Road, Manchester M13 9WL, UK. ${ }^{6}$ Division of Informatics, Imaging \& Data

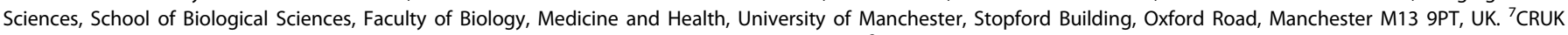
Manchester Institute, The University of Manchester, Alderley Park, Alderley Edge SK10 4TG, UK. ${ }^{8}$ Department of Clinical Biochemistry, Manchester University NHS Foundation Trust, Manchester Academic Health Science Centre, Oxford Road, Manchester M13 9WL, UK. 'Department of Obesity Medicine, Diabetes \& Endocrinology, Salford Royal NHS

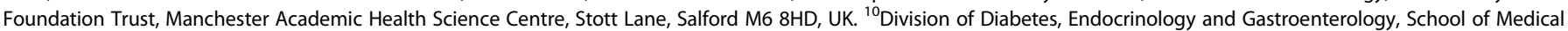
Sciences, Faculty of Biology, Medicine \& Health, University of Manchester, Oxford Road, Manchester M13 9PL, UK. ${ }^{11}$ Department of Surgery, Salford Royal NHS Foundation Trust,

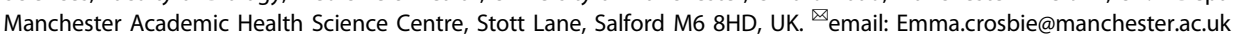

Received: 18 March 2021 Revised: 10 November 2021 Accepted: 16 November 2021

Published online: 2 December 2021 
postmenopausal and premenopausal anovulatory women, promotes endometrial proliferation and tumourigenesis [8]. Other contributors include metabolic dysfunction, insulin resistance and hyperinsulinaemia, factors that further stimulate endometrial growth through direct and indirect pathways [9]. Obesity is a chronic pro-inflammatory state, with increased circulating levels of the inflammatory markers C-reactive protein (CRP), interleukin- 6 (IL-6) and tumour necrosis factor-alpha (TNF-a) [10]. These inflammatory markers may mediate alterations in the immune microenvironment of the endometrium that support neoplastic transformation.

Weight loss achieved and sustained through bariatric surgery or dietary intervention has been shown to reduce endometrial cancer risk [11-14]. In a prospective study of women undergoing gastric bypass or sleeve gastrectomy-induced weight loss, we found occult endometrial cancer or its precursor lesion, atypical hyperplasia, in $14 \%$ of those sampled at baseline [15]. Spontaneous resolution of atypical hyperplasia was observed 2 months following bariatric surgery in $3 / 6$ women and a further 2/6 were successfully managed with intrauterine progestin. In those with histologically normal endometrium, we observed a weight lossassociated down-regulation of pro-proliferative signalling pathways, including reductions in proliferation marker Ki-67. These findings support the hypothesis that weight loss instigates changes in the endometrium that influence its susceptibility to malignant transformation. We propose that the immune microenvironment is a dynamic regulator of endometrial health that is perturbed in obesity and restored with weight loss. The aim of this study was to measure the impact of obesity and weight loss on the immunological landscape of the endometrium using matched serum and endometrial tissue samples from our cohort of women undergoing intentional weight loss.

\section{METHODS \\ Study participants}

This was a prospective cohort study of women with morbid obesity (BMI $\geq$ $40 \mathrm{~kg} / \mathrm{m}^{2}$ ) undergoing weight loss management at Salford Royal NHS Foundation Trust [15]. Approval was obtained from the North West Research Ethics Committee (12/NW/0050) and the study was prospectively registered (ISRCTN17241389). All participants gave written, informed consent. Height was measured using a stadiometer, weight was measured using electronic scales after removal of bulky clothing, and BMI derived $\left(\mathrm{kg} / \mathrm{m}^{2}\right)$. Blood and endometrial tissue were collected at baseline, and two and 12 months following medically-supervised low-calorie diet or bariatric surgery, either gastric bypass, sleeve gastrectomy or gastric banding. Postmenopausal status was defined as last menstrual bleed $>12$ months previously. Premenopausal participants were sampled in the late proliferative phase, except for baseline biopsies of women undergoing bariatric surgery, which were obtained under general anaesthetic. Endometrial sampling was by Pipelle ${ }^{\Theta}$ (Carefusion, UK) or MedGyn Endosampler $^{\circ}$ (MedGyn, Illinois, USA). Tissue was fixed in $10 \%$ neutral buffered formalin prior to embedding in paraffin wax. Histopathological interpretation of haematoxylin and eosin-stained sections was by specialist gynaecological pathologists (RJM and JB) according to the WHO classification system $[16,17]$. Serum IL-6 and TNF-a were measured using a DuoSet ELISA development kit (R\&D Systems, Abingdon, UK) and high sensitivity CRP (hsCRP) was measured by an in-house antibody sandwich ELISA technique with antihuman CRP primary antibodies from Abcam (Cambridge, UK).

\section{Immunohistochemistry}

Four-micron sections were cut from formalin-fixed paraffin-embedded tissue samples and multiplex immunofluorescent staining was performed using a Ventana Autostaining Robot (Ventana Medical Systems, Arizona, USA), with automation of deparaffinisation, antigen retrieval and incubation but manual application of reagents. Antibodies (Table S1) were applied sequentially in the following order, with fluorescent disclosure of each antibody with a different fluorophore, and antibody denaturation between each primary antibody application: CD8, CD68, CD3, FOXP3, CD56, PD-1. Following multiplex staining, all sections were cover-slipped with the Prolong aqueous mounting agent (Thermo Fisher, Massachusetts, USA) that also contained DAPI for counter-staining. The detailed multiplex staining protocol is given in Supplementary Methods.

\section{Tissue imaging and analysis}

Stained sections were captured by slide scanning and image acquisition using the Vectra 3.05 multispectral imager (PerkinElmer, Massachusetts, USA). Each section was first scanned at a magnification of $10 x$, producing a low-resolution overview of the tissue. Up to 10 regions of interest were randomly sampled from the low-resolution overview image, using Phenochart whole slide viewer (PerkinElmer, Massachusetts, USA). These regions were then scanned at a higher magnification of $\times 20(0.496 \mu \mathrm{m} /$ pixel). Each scanned region consisted of nine multispectral images (MSI) that were automatically stitched together.

MSI were unmixed and analysed in three stages: spectral unmixing, cell segmentation and cell phenotyping. Linear spectral unmixing was carried out using InForm 2.4 software (PerkinElmer, Massachusetts, USA). For unmixing, a spectral library was built comprising individual fluorophore spectra. Each spectrum was acquired from slides that were single stained for each antibody, using the same experimental parameters as the multiplex experiment. A slide stained with DAPI only was used to extract the DAPI spectrum. A slide that underwent all steps in the multiplex experiment without application of antibodies or fluorophores was used to extract the spectrum of tissue auto-fluorescence. After spectral unmixing, six resultant images were generated representative of the staining intensities of each fluorophore (Fig. 1) in which the intensity at each pixel is proportional to the quantity of fluorophore and its corresponding epitope, at that pixel.

Cell segmentation was conducted using Inform software. A two-step process was carried out using only the DAPI channel. First, nuclear components were detected using nuclear stain intensity. Then the cytoplasm around each nucleus was simulated by cell expansion of $2 \mu \mathrm{m}$ and measurements generated for marker intensity in different compartments (mean, minimum, maximum and standard deviation of intensity in cytoplasm or nucleus).

Cell scoring was carried out using an in-house software developed in the imaging department of the University of Manchester [18]. The software automatically determines if a cell is positive or negative for each stain using a pre-determined threshold cut-off. Positivity for each cell type was determined by the intensity of each marker in the primary cell compartment where it was usually expressed. In our study, markers were cytoplasmic or membranous. Before cell scoring, the intensity of each marker was re-scaled onto a greyscale colour map, where the brightest and darkest values corresponded to the $99 \%$ and $1 \%$ percentiles of the marker's pixel intensities in the full dataset. Having a consistent colour-map per marker ensured that the same intensity value was represented with equal brightness in all images.

Guided by a pathologist (RB), a single threshold for each marker was selected as a cut-off to determine positivity across the entire dataset. The threshold was identified by its ability to separate positive from negative cells in a set of 20 regions of interest from different patients. The resultant number of positive cells per unit area was measured in each of the patient samples for each of the antigens assayed.

\section{Statistical analysis}

All statistical analyses were conducted in R 3.5.1 ×64/RStudio 1.0.143×64. To determine suitability of data for parametric testing, QQ normality plots were called for both response variables and response variable linear model residuals vs. standardised residuals for the six tissue immune markers (CD8, CD68, CD3, FOXP3, CD56, PD-1), three serum inflammatory markers (hsCRP, IL-6, TNF-a) and clinical parameters (BMI and weight). Only BMI and weight held distributions approaching normality. Homogeneity of variance was probed by Barlett's Test followed by Levene's Test. Fligner-Killeen tests were used for variables determined to have departures from normality. All variables except for FOXP3, CRP, TNF-a and BMI demonstrated heteroscedastic variances.

Normality and variance violations prohibited parametric analysis of data, but only $7 \%$ of observations were suitable for BoxCox transformation into normal distributions. Kruskal-Wallis tests were therefore used to determine if there were any significant changes in immune, inflammatory and clinical biomarkers between baseline and 2 months, and between baseline and 12 months. Dunn's multiple comparisons post hoc was implemented to determine groups with significant differences with a Sidak or Benjamini Hochberg multiple comparisons adjustment.

Time series correlation modelling explored relationships between tissue and serum biomarkers. Nonlinear mixed effect (n/me) modelling was 

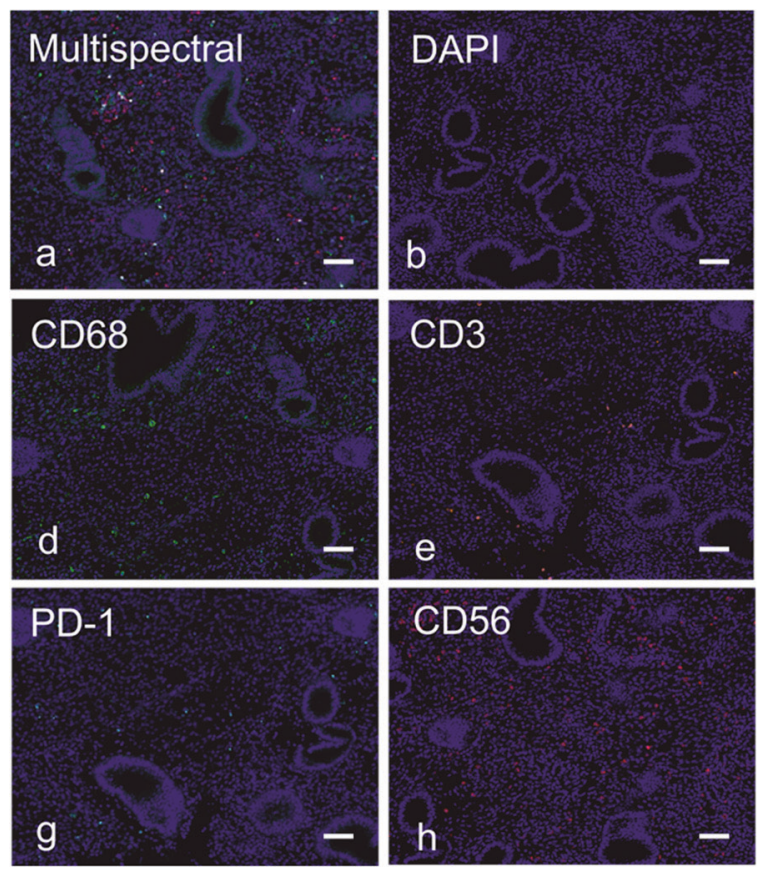

Fig. 1 Multiplex immunofluorescence and automated image analysis to measure tumour infiltrating immune cells. Each of (a-h) correspond to the same tissue region, demonstrating the raw unmixed multispectral image containing all immunofluorescent stains (a) and resultant separately resolved cellular populations after spectral unmixing in (c-h), specifically in (c) CD8+ cells, (d) CD68+ cells, (e) CD3 + cells, (f) FOXP3 + cells, (g) PD-1+ cells, and (h) CD56 + cells; DAPI nuclear counterstain shown in (b) was used for nuclear segmentation and resultant cellular segmentation; magnification $\times 200$ in all panels and scale bar 100 microns. All markers are displayed using pseudo-colours.

Table 1. Systemic inflammatory markers in matched serum samples.

\begin{tabular}{|c|c|c|c|c|c|}
\hline Serum biomarker & Baseline (median) & 2 months (median) & 12 months (median) & Baseline - 2 months & Baseline - 12 months \\
\hline CRP $(\mu \mathrm{g} / \mathrm{ml})$ & 5.9 & 4.2 & 1.7 & $p=0.5572$ & $p<0.0086^{a}$ \\
\hline IL-6 (pg/ml) & 4.8 & 1.7 & 1.2 & $p=0.0021^{a}$ & $p<0.0001^{a}$ \\
\hline TNF- $\alpha(p g / m l)$ & 29.1 & 31.4 & 25.2 & $p=0.7307$ & $p=0.8609$ \\
\hline
\end{tabular}

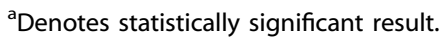

Bold values indicates statistically significant $p<0.05$ values.

trialled to accommodate distribution differences, unequal class sizes, autocorrelation, and heteroscedastic variances; however, not all markers successfully converged using this method. Repeated measures correlation modelling was therefore used since it protects against class imbalance, correlated error rates and heteroscedasticity. To alleviate requirements for normally distributed data we bootstrapped variable-specific distributions during model generation.

\section{RESULTS}

\section{Study participants}

From a total cohort of 80 participants, 43 had matched serum and tissue samples at baseline, and 2 months and 12 months after weight loss intervention and were included in this study. Their median age and BMI at baseline were 44 years (range 24-60 years) and $52 \mathrm{~kg} / \mathrm{m}^{2}$ (range $38-69 \mathrm{~kg} / \mathrm{m}^{2}$ ), respectively. Most (37/43, $86.0 \%)$ were premenopausal. Thirty-seven women underwent bariatric surgery, either gastric bypass (26/37, 70.3\%), sleeve gastrectomy $(11 / 37,29.7 \%)$ or gastric banding $(1 / 37,2.7 \%)$, and $6 / 43$ (14\%) underwent medically-supervised low-calorie diet. At baseline, six women had occult atypical endometrial hyperplasia, five of which resolved with weight loss and/or intrauterine progestin, as previously described [15]. Median weight loss was greater for women who underwent bariatric surgery compared to those who followed a low-calorie diet, both at 2 months [-15.1 kg (MAD 4.59, $p=0.0201$ ) vs. $-1.7 \mathrm{~kg}$ (MAD $2.48 \mathrm{~kg}, p=0.936$ ) respectively] and 12 months [-63.3 kg (MAD 17.64, $p<0.0001$ ) vs. $-13.0 \mathrm{~kg}$ (MAD 11.1, $p=0.0679$ ) respectively]. Median BMI was $53 \mathrm{~kg} / \mathrm{m}^{2}$ and $47.1 \mathrm{~kg} / \mathrm{m}^{2}$ at baseline, $45 \mathrm{~kg} / \mathrm{m}^{2}(p=0.0002)$ and $46.7 \mathrm{~kg} / \mathrm{m}^{2}(p=0.936)$ at 2 months and $35 \mathrm{~kg} / \mathrm{m}^{2}(p<0.0001)$ and $43.6 \mathrm{~kg} / \mathrm{m}^{2}(p=0.201)$ at 12 months in women undergoing bariatric surgery and low-calorie diet, respectively.

Systemic inflammatory markers CRP, IL- 6 and TNF- $a$ were mildly elevated at baseline (Table 1). Median CRP was significantly lower at 12 months ( $p=0.0086$ ) compared to baseline (Fig. 2). CRP correlated significantly with weight loss $\left(p=3.62 \times 10^{-6}, r=0.570\right)$ and BMI reduction ( $p=0.00014, r=0.491$ ) (Fig. 3). Median IL-6 showed significant reductions at 2 months $(p=0.0021)$ and 12 months $(p<$ 0.00001 ) compared to baseline (Fig. 2), which correlated with weight loss $(p=0.00032, r=0.459)$ and BMI reduction $\left(p=3.15 \times 10^{-5}, r=\right.$ 0.53 ) (Fig. 3). TNF-a levels did not change significantly over time, with weight loss or with $\mathrm{BMI}$ reduction.

The density of endometrial CD56, CD68, CD3, FOXP3, CD56 and PD1-positive immune cells at baseline, 2 months and 12 months after weight loss intervention is shown in Table 2 and Fig. 2 . The density of CD8 + immune cells was higher at 2 months and 12 months compared to baseline (Table 2) and inversely correlated with weight 
Time Point Baseline 卢 2 Months 卢 12 Months
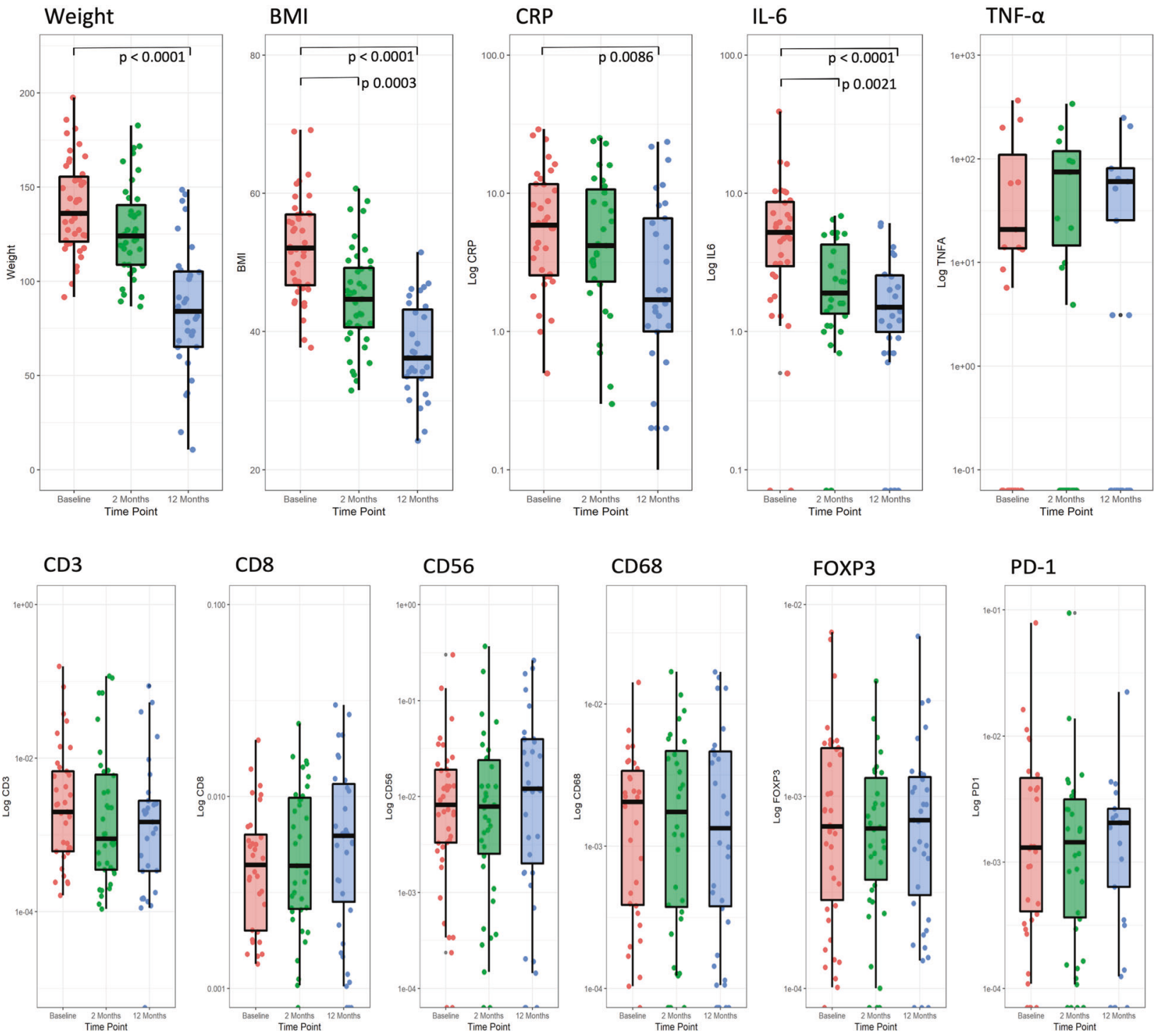

Fig. 2 Change in clinical, inflammatory and immune biomarkers with time. Box and jitter plots for biomarkers across time points, including weight, BMI, systemic inflammatory and immune cell marker densities. $Y$ axes are log scale. Significant $p$ values are provided at bracketed comparators where relevant (Kruskal-Wallis tests with Dunn-Šidák corrections). The $\alpha$ for all tests was 0.05. Significant reductions were observed for weight between baseline: 12 months $(p<0.0001)$; BMI between baseline: 2 months $(p=0.0003)$ and baseline: 12 months ( $p<$ $0.0001)$; CRP between baseline: 12 months $(p=0.0086)$; and for IL-6 baseline: 2 months $(p=0.0021)$ and baseline: 12 months $(p<0.0001)$.

$(p=0.0097, r=-0.323)$ and BMI $(p=0.0093, r=-0.325)$ (Fig. 3). The density of CD68+, FOXP3+, PD-1+ and CD56+ immune cells showed no significant change with time (Table 2, Fig. 2) nor correlation with weight loss or BMI. The density of CD3+immune cells was inversely correlated with systemic IL-6 levels ( $p=0.037 ; r=$ -0.318) (Table 3, Fig. 4). No significant associations were seen between the other markers (Table 3 ). Positive associations between CD3 and CD68-positive immune cell infiltrates and systemic CRP levels did not reach statistical significance; neither did the positive association between CD68+ immune cells and systemic TNF-a levels.

\section{DISCUSSION}

Obesity increases endometrial cancer risk and weight loss is protective, but the underlying mechanisms are incompletely understood. Here, multiplex fluorescence immunohistochemistry was used to quantify and phenotype immune cells in the endometrium before and after weight loss in women with class III obesity. There was a significant rise in CD8+immune cell density at 2 and 12 months that correlated with weight loss. We also found significant reductions in systemic inflammatory markers CRP and IL-6 with weight loss, the latter inversely correlated with $\mathrm{CD} 3+$ immune cell density in the endometrium. Taken together, these findings indicate that the immunological landscape of the endometrium may be affected by obesity and weight loss, and by extension, that the immune microenvironment may influence susceptibility to neoplastic change.

Previous work has shown a strong positive correlation between serum levels of pro-inflammatory cytokines and adipokines and endometrial cancer risk [19]. In a prospective study of 107 women sampled before and after bariatric surgery, Linkov et al. (2017) reported near normalisation of serum CRP and IL-6 levels, 
a

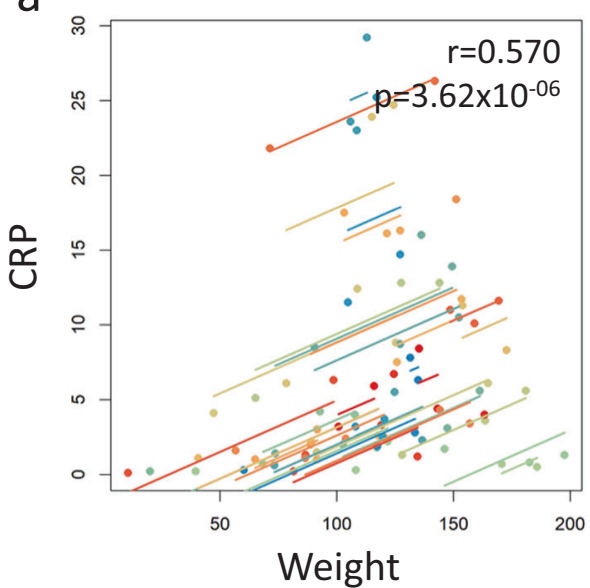

C

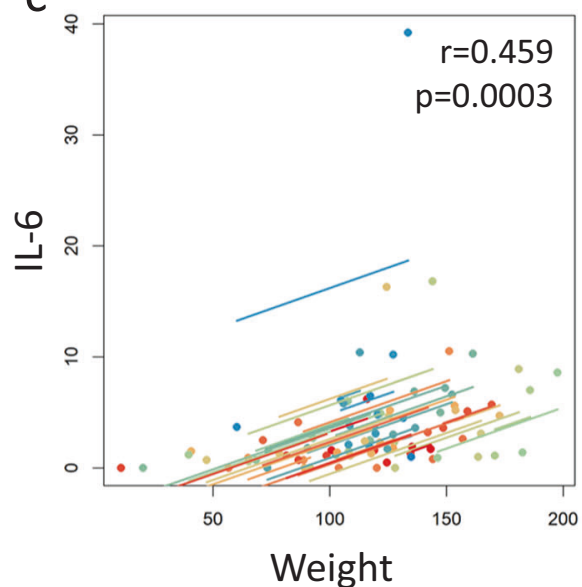

e

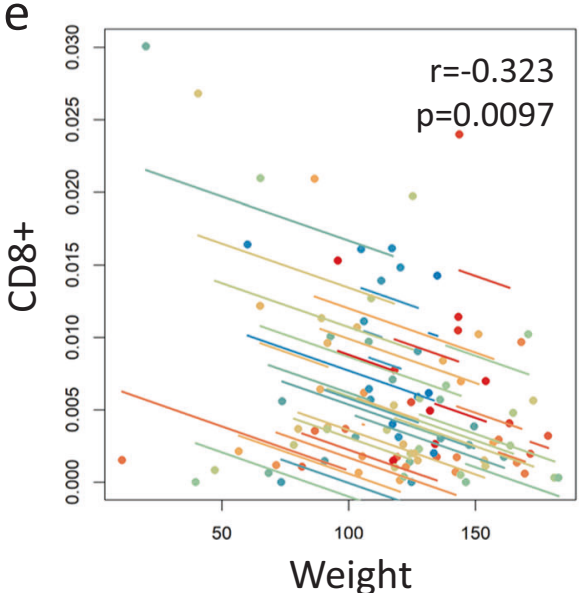

b
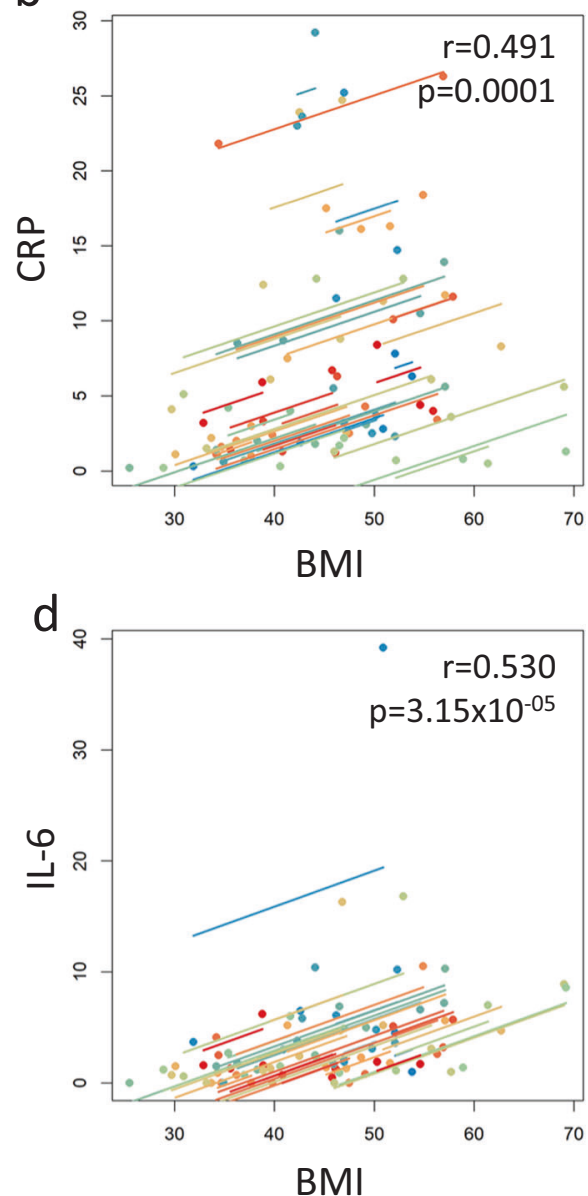

f

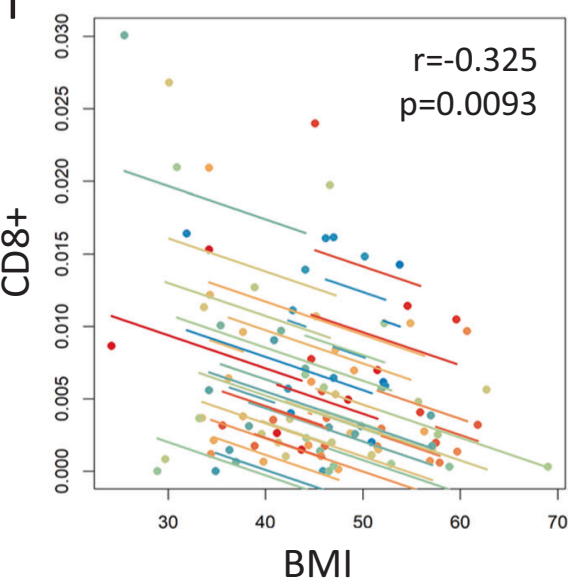

Fig. 3 Correlations between weight, BMI, inflammatory biomarkers and immune infiltrates. Repeated measures correlation plots are shown. Significant positive correlations were observed between reduced systemic inflammatory marker CRP and reduced weight (a) or BMI (b) $\left(r=0.57, p=3.62 \times 10^{-6}\right.$ and $r=0.491, p=0.0001$, respectively). Significant positive correlations were observed between reduced systemic inflammatory marker IL-6 and reduced weight c) or BMI (d) $\left(r=0.459, p=0.0003\right.$ and $r=0.530, p=3.15 \times 10^{-5}$, respectively). Significant negative correlations were observed between increased CD8+ immune cells and reduced weight (e) or BMI (f) $(r=-0.323, p=0.0097$ and $r=$ $-0.325, p=0.0093$, respectively). Repeated measures correlation distributions were bootstrapped from data structures to accommodate potential unequal variance and distributions within the time dependent analysis. The $\alpha$ for all tests was 0.05 .

amongst other pro-inflammatory biomarkers, and concluded that their change may reflect the protective effect of weight loss on endometrial cancer risk [20]. We found one previous study investigating the impact of obesity and weight loss on the immunological landscape of the endometrium [21]. In paired endometrial samples before and 12 months after bariatric surgery-induced weight loss, the authors found no significant difference in $\mathrm{CD} 3+$ or $\mathrm{CD} 20+$ immune cell density on tissue 
Table 2. Endometrial immune cell infiltrates in matched tissue samples.

\begin{tabular}{llllll}
$\begin{array}{l}\text { Tissue immune cell } \\
\text { biomarker }\end{array}$ & $\begin{array}{l}\text { Baseline Median \% } \\
\text { positive cells }\end{array}$ & $\begin{array}{l}\mathbf{2} \text { months Median \% } \\
\text { positive cells }\end{array}$ & $\begin{array}{l}\mathbf{1 2} \text { months Median \% } \\
\text { positive cells }\end{array}$ & $\begin{array}{l}\text { Baseline - } \\
\mathbf{2} \text { months }\end{array}$ & $\begin{array}{l}\text { Baseline - } \\
\mathbf{1 2} \text { months }\end{array}$ \\
\hline CD56 & 0.0071 & 0.0069 & 0.0116 & $p=0.7978$ & $p=0.7737$ \\
\hline CD68 & 0.0019 & 0.0012 & 0.0008 & $p=0.7490$ & $p=0.6634$ \\
\hline CD3 & 0.0019 & 0.0009 & 0.0013 & $p=0.3485$ & $p=0.1717$ \\
\hline CD8 & 0.0037 & 0.0038 & 0.0056 & $p=0.3074$ & $p=0.3016$ \\
\hline FOXP3 & 0.00069 & 0.00069 & 0.00066 & $p=0.6672$ & $p=0.6445$ \\
\hline PD-1 & 0.0011 & 0.0008 & 0.0012 & $p=0.5822$ & $p=0.4360$ \\
\hline $\begin{array}{l}\text { Median total number of } \\
\text { cells counted/sample }\end{array}$ & 14,675 & 7966 & 9749 & Not applicable & \\
\hline
\end{tabular}

Table 3. Correlation of systemic inflammatory markers with endometrial immune cell infiltrates.

\begin{tabular}{|llll|}
\hline & CRP & IL-6 & TNF- $\boldsymbol{a}$ \\
\hline CD8+ & 0.7720 & 0.1658 & 0.3747 \\
\hline CD68+ & 0.1852 & 0.4618 & 0.1473 \\
\hline CD3+ & 0.2539 & $\mathbf{0 . 0 3 7 5}$ & 0.48457 \\
\hline FOXP3+ & 0.8527 & 0.7855 & 0.7209 \\
\hline CD56+ & 0.6401 & 0.9321 & 0.4723 \\
\hline PD-1+ & 0.5295 & 0.8424 & 0.8989 \\
\hline
\end{tabular}

Table shows $p$ values.

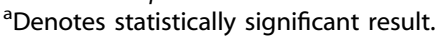

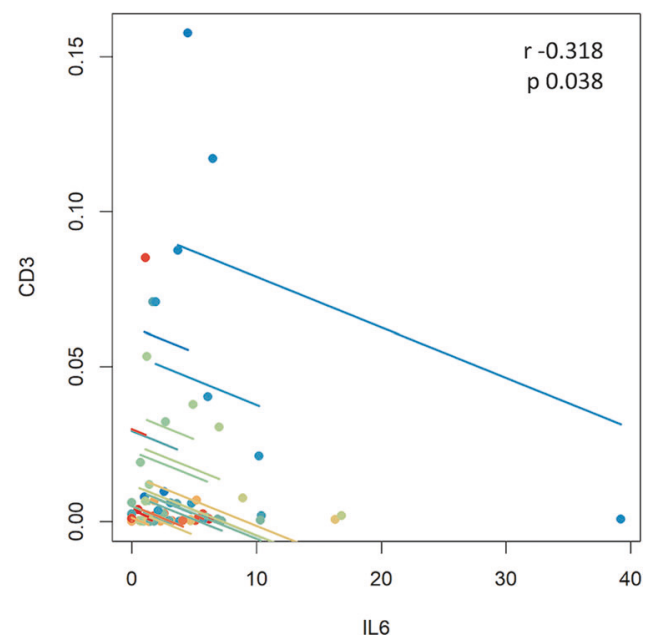

Fig. 4 Inverse correlation of CD3 + immune cell infiltrate density with IL- 6 across time points. A repeated measures correlation plot of Log CD3+immune cell density vs. IL- 6 intensity. A significant inverse correlation was observed ( $p=0.038, r=-0.318$ ). Repeated measures correlation distributions were bootstrapped from data structure to accommodate potential unequal variance and distributions within the time dependent analysis. The $\alpha$ for all tests was 0.05 .

microarrays for a subset of their overall study population. They did not measure changes in macrophage (CD68), natural killer cell (CD56), cytotoxic $\mathrm{T}$ cell (CD8), regulatory $\mathrm{T}$ cell (FOXP3) or programmed cell death protein 1 (PD-1) expressing immune cell populations and were therefore not able to make firm conclusions as to the impact of obesity and weight loss on the endometrial immune microenvironment.

Here, we show convincing evidence of a change in the immunological landscape of morphologically normal endometrium in women with class III obesity who undergo significant weight loss. The reduction in systemic inflammation that accompanies weight loss is associated with a recruitment of protective immune cell types to the endometrium, in particular CD8+ cytotoxic T cells. This is consistent with previous work comparing circulating immune cell populations in obese and lean study participants that found higher $\mathrm{CD} 8+\mathrm{T}$ cell counts in lean individuals [22]. CD8+T-cells are essential mediators of cancer immune surveillance, keeping tissues healthy through the recognition and selective elimination of cells expressing cancerspecific antigens [23]. An influx of these highly specialised anticancer immune cells to the endometrium with weight loss suggests they may have a role in restoring endometrial health and protecting against endometrial cancer. It is already well established that high volume tumour infiltrating CD8+lymphocytes portend good survival outcomes from colorectal [24], melanoma [25] and many other tumour types [26], including endometrial cancer $[27,28]$. Extrapolating these findings to a role for CD8+ $\mathrm{T}$ cells in maintaining endometrial health, restoring health through risk-reducing interventions e.g. weight loss, and preventing cancer is an appealing concept worthy of further investigation. It is interesting that systemic IL-6 levels were inversely correlated with endometrial $\mathrm{CD} 3+\mathrm{T}$ cells since the cancer-promoting properties of IL- 6 are well known. It has been shown to stimulate growth, DNA methylation and metastasis and to destabilise the immune microenvironment [29]. Its reduction with weight loss may therefore encourage the recruitment of $\mathrm{CD} 3+$ and other protective immune cells to the endometrium. A favourable immunological landscape may enable the natural clearance of latent endometrial precursor and pre-cancer lesions, protecting endometrial health and preventing endometrial cancer [30].

This is the first study, to our knowledge, that compares immune biomarkers in serum and endometrial tissue before and after weight loss in women with class III obesity. Serial measurements across three time points add to the strength of the findings and distinguish short- and long-term effects of weight loss. It is interesting that changes to blood and tissue immune biomarkers were observed as early as 2 months after weight loss intervention, before the majority of excess body weight had been shed. Changes to the immune microenvironment coincided with the natural clearance of atypical hyperplasia in 3/6 women with occult endometrial abnormalities at baseline, indicating their potential role in the restoration of endometrial health. Indeed, resolution of 5/6 endometrial abnormalities occurred by 6 months post-bariatric surgery, before women had achieved a healthy body weight. Immune cell infiltrates vary with menstrual cycle phase and menopausal status [31], and this was controlled for where possible. Multiplex immunofluorescence labelling enabled simultaneous quantification and phenotyping of immune cell markers using a single tissue section. This is advantageous for analysis of small biopsy samples and enables an appreciation of the interplay between different immune cell types within different tissue compartments.

A limitation of our work is the relatively small sample set; however, it derives from a larger cohort of 80 participants and 
highlights the challenge of longitudinal studies involving sequential invasive sampling. We were unable to control for potential confounding factors, particularly age and menopausal status, and the numbers were too small to allow subgroup analysis by weight loss intervention. Although interesting, our findings are correlative and their significance is uncertain. We were limited by the number of fluorophores we could multiplex in one experiment, precluding the detailed phenotyping of immune cell sub-populations, for example $M 1$ and $M 2$ macrophages. Endometrial recruitment of protective $\mathrm{M} 2$ macrophages with weight loss may have been masked by the simultaneous loss of pro-inflammatory M1 macrophages, for instance, and our study design did not allow these cellular sub-populations to be differentiated from each other. Other immune cells may also be important in regulating endometrial health and more research is needed.

If validated in larger studies, the insights provided here could have important clinical implications. Immune biomarkers could enable individualised endometrial cancer risk prediction and also measure the success of risk-reducing interventions. Research could be directed towards encouraging recruitment of protective immune cells to the endometrium to boost the natural clearance of precursor and precancerous lesions. We urgently need innovative solutions to avert the impending surge of endometrial cancer diagnoses predicted by the escalating global obesity problem.

\section{REFERENCES}

1. Cancer Research UK. Uterine Cancer Statistics. http://www.cancerresearchuk.org/ health-professional/cancer-statistics/statistics-by-cancer-type/uterine-cancer.

2. Crosbie EJ, Zwahlen M, Kitchener HC, Egger M, Renehan AG. Body Mass Index, Hormone Replacement Therapy, and Endometrial Cancer Risk: A Meta-Analysis. Cancer Epidemiol Biomarkers Prev. 2010;19:3119.

3. Kitson SJ, Crosbie EJ. Endometrial Cancer and Obesity. Obstetrician Gynaecologist. 2019;21:237-45.

4. Kelly T, Yang W, Chen CS, Reynolds K, He J. Global burden of obesity in 2005 and projections to 2030. Int J Obes (Lond). 2008;32(Sep):1431-7.

5. Crosbie E, Morrison J. The emerging epidemic of endometrial cancer: Time to take action. Tovey D, editor. The Cochrane database of systematic reviews. 2014;12:ED000095.

6. Lu KH, Broaddus RR. Endometrial Cancer. N Engl J Med. 2020;383:2053-64.

7. MacKintosh ML, Crosbie EJ. Prevention Strategies in Endometrial Carcinoma. Curr Oncol Rep. 2018;20:101.

8. Kaaks R, Lukanova A, Kurzer MS. Obesity, endogenous hormones, and endometrial cancer risk: a synthetic review. Cancer Epidemiol Biomark Prev. 2002;11:1531-43.

9. Khandekar MJ, Cohen P, Spiegelman BM. Molecular mechanisms of cancer development in obesity. Nature Rev Cancer. 2011;11:886-95.

10. Modugno F, Ness RB, Chen C, Weiss NS. Inflammation and endometrial cancer: a hypothesis. Cancer Epidemiol Biomarkers Prev. 2005;14:2840-7.

11. Anveden A, Taube M, Peltonen $M$, Jacobson $P$, Andersson-Assarsson JC, Sjoholm $\mathrm{K}$, et al. Long-term incidence of female-specific cancer after bariatric surgery or usual care in the Swedish Obese Subjects Study. Gynecol Oncol. 2017;145:224-9.

12. Luo J, Chlebowski RT, Hendryx M, Rohan T, Wactawski-Wende J, Thomson CA et al. Intentional Weight Loss and Endometrial Cancer Risk. J Clin Oncol. 2017;35:1189-93.

13. Look AHEAD Research Group, Yeh HC, Bantle JP, Cassidy-Begay M, et al. Intensive Weight Loss Intervention and Cancer Risk in Adults with Type 2 Diabetes: Analysis of the Look AHEAD Randomized Clinical Trial. Obesity (Silver Spring). 2020;28:1678-86.

14. Barr CE, Ryan NAJ, Derbyshire $A E$, Wan $Y L$, MacKintosh $M L$, McVey RJ, et al. Weight Loss During Intrauterine Progestin Treatment for Obesity-associated Atypical Hyperplasia and Early-Stage Cancer of The Endometrium. Cancer Prev Res (Phila). 2021;14:1041-50.

15. MacKintosh ML, Derbyshire AE, McVey RJ, Bolton J, Nickkho-Amiry M, Higgins $C L$, et al. The impact of obesity and bariatric surgery on circulating and tissue biomarkers of endometrial cancer risk. Int J Cancer. 2019;144:641-50.

16. Emons G, Beckmann MW, Schmidt D, Mallmann P. Uterus commission of the Gynecological Oncology Working Group (AGO). New WHO Classification of Endometrial Hyperplasias. Geburtshilfe Frauenheilkd. 2015;75:135-6.

17. Kurman RJ, Carcangiu M, Herrington CS, Young. WHO classification of tumours of female reproductive organs. 4th edition. Lyon: IARC; 2014.
18. Tsakiroglou AM, Fitzpatrick S, Nelson L, West C, Linton K, Zeng K, et al. Quantifying cell-type interactions and their spatial patterns as prognostic biomarkers in follicular lymphoma. Proc. SPIE10581, Medical Imaging 2018: Digital Pathology, 105810G 2018; https://doi.org/10.1117/12.2293572.

19. Fortner RT, Hüsing A, Kühn T, Konar M, Overvad K, Tjønneland A, et al. Endometrial cancer risk prediction including serum-based biomarkers: results from the EPIC cohort. Int J Cancer. 2017;140:1317-23.

20. Linkov F, Goughnour SL, Ma T, Xu Z, Edwards RP, Lokshin AE, et al. Changes in inflammatory endometrial cancer risk biomarkers in individuals undergoing surgical weight loss. Gynecol Oncol. 2017;147:133-8.

21. Linkov F, Elishaev E, Gloyeske N, Edwards R, Althouse AD, Geller MA, et al. Bariatric surgery-induced weight loss changes immune markers in the endometrium of morbidly obese women. Surg Obes Relat Dis. 2014;10:921-6.

22. El-Hafez HA, El-Aziz SMA. Association between immune competence and metabolic parameters in obesity. Trend Med Res. 2012;7:53-61.

23. Dunn GP, Old LJ, Schreiber RD. The Three Es of Cancer Immunoediting. Annu Rev Immunol. 2004;22:329-60.

24. Galon J, Costes A, Sanchez-Cabo F, Kirilovsky A, Mlecnik B, Lagorce-Pagès C, et al Type, density, and location of immune cells within human colorectal tumors predict clinical outcome. Science. 2006;313:1960-4.

25. Maibach F, Sadozai H, Seyed Jafari SM, Hunger RE, Schenk M. Tumor-Infiltrating Lymphocytes and Their Prognostic Value in Cutaneous Melanoma. Front Immunol. 2020;11:2105.

26. Fridman WH, Pagès $F$, Sautès-Fridman $C$, Galon J. The immune contexture in human tumours: impact on clinical outcome. Nat Rev Cancer. 2012;12:298-306.

27. Guo F, Dong Y, Tan Q, Kong J, Yu B. Tissue Infiltrating Immune Cells as Prognostic Biomarkers in Endometrial Cancer: A Meta-Analysis. Dis Markers. 2020:2020:1805764.

28. Workel HH, Komdeur FL, Wouters MC, Plat A, Klip HG, Eggink FA, et al. CD103 defines intraepithelial CD8 + PD1 + tumour-infiltrating lymphocytes of prognostic significance in endometrial adenocarcinoma. Eur J Cancer. 2016;60:1-11.

29. Weber R, Groth C, Lasser S, Arkhypov I, Petrova V, Altevogt P, et al. IL-6 as a major regulator of MDSC activity and possible target for cancer immunotherapy. Cell Immunol. 2021;359:104254.

30. Mackintosh ML, Crosbie EJ. Obesity-driven endometrial cancer: is weight loss the answer? BJOG. 2013;120:791-4.

31. Berbic M, Fraser IS. Immunology of normal and abnormal menstruation. Womens Health (Lond). 2013;9:387-95.

\section{ACKNOWLEDGEMENTS}

We acknowledge the generous gift of time and samples from the women in our study. We would like to thank Hayley Ammori, Joe Woods, Tina Pritchard, Donna Roberts, Adele Poole and Linsey Nelson for their help with patient recruitment, data acquisition, data management and obtaining regulatory approvals. We are grateful for the invaluable assistance of Gill Hesketh who sadly passed away before our study was completed.

\section{AUTHOR CONTRIBUTIONS}

EJC was Principal Investigator, obtained funding for the study and is its guarantor EJC and RB conceptualised the study and supervised its execution. MLM, AED, AAS and BJA contributed to patient recruitment, sample acquisition and weight management. RJM, JB and PWP performed pathological interpretation and serum analyses. AN performed laboratory analyses, interpreted the results and wrote first draft of the paper. SB and GA supported image acquisition and analysis. AT, MF and TW contributed to data interpretation and statistical analyses. RB and EJC assisted data interpretation and paper writing. All authors provided critical comment and approved the final version of the paper.

\section{FUNDING}

EJC was supported through an National Institute for Health Research (NIHR) Clinician Scientist award (NIHR-CS-012-009). MLM and AED were supported by Manchester University NHS Foundation Trust Clinical Research Fellowships. This article presents independent research funded by the NIHR, supported by the NIHR Manchester Biomedical Research Centre (IS-BRC-1215-20007) and facilitated by the Greater Manchester Local Clinical Research Network. The views expressed are those of the authors and not necessarily those of the NHS, the NIHR or the Department of Health The funder had no role in the design and conduct of the study; collection, management, analysis, and interpretation of the data; preparation, review, or approval of the study; and decision to submit the study for publication. 


\section{COMPETING INTERESTS}

The authors declare no competing interests.

\section{ADDITIONAL INFORMATION}

Supplementary information The online version contains supplementary material available at https://doi.org/10.1038/s41366-021-01027-6.

Correspondence and requests for materials should be addressed to Emma J. Crosbie.

Reprints and permission information is available at http://www.nature.com/ reprints

Publisher's note Springer Nature remains neutral with regard to jurisdictional claims in published maps and institutional affiliations.
Open Access This article is licensed under a Creative Commons Attribution 4.0 International License, which permits use, sharing, adaptation, distribution and reproduction in any medium or format, as long as you give appropriate credit to the original author(s) and the source, provide a link to the Creative Commons license, and indicate if changes were made. The images or other third party material in this article are included in the article's Creative Commons license, unless indicated otherwise in a credit line to the material. If material is not included in the article's Creative Commons license and your intended use is not permitted by statutory regulation or exceeds the permitted use, you will need to obtain permission directly from the copyright holder. To view a copy of this license, visit http://creativecommons. org/licenses/by/4.0/.

(c) The Author(s) 2021 